\title{
Preinvasive carcinoma of the cornea and conjunctiva
}

\author{
A. J. DARK AND B. W. STREETEN \\ From the Department of Ophthalmology, Veterans Administration Hospital, and the \\ Departments of Ophthalmology and Pathology, Upstate Medical Center, \\ Syracuse, New York 13210, USA
}

SUMMARY Greyish-white spots, varying in size, caused diagnostic problems in 2 patients with preinvasive corneal carcinoma. Keratectomy specimens permitting light and electron microscopy indicated that the smaller spots predominating in one patient correlated with epithelial microcysts and vacuoles, while areas of parakeratosis accounted for the macroscopic white patches found in the other. A variety of ultrastructural abnormalities, including excessive basement membrane fibrillogenesis, were present in both cases.

When carcinoma-in-situ involves the cornea it usually appears as a greyish (rarely pigmented) gelatinous elevation extending from the limbus on to the cornea. In contrast the 2 patients reported here presented with a translucent film-like change of the central epithelium in which white dots, microscopic in the first patient but visible to the naked eye in the second, were present. These somewhat unusual clinical findings, together with correlated light and electron microscopic appearances, have prompted this report.

\section{Case reports}

CASE 1

A 29-year-old white male was first seen in May 1971 , at age 22, complaining of blurred vision in his left eye, which was irritable and watered, especially when reading. His right eye was normal apart from a refractive amblyopia and a small biopsyconfirmed squamous papilloma of the outer canthus. Visual acuity of the left eye corrected to $20 / 40$, though several weeks previously it had been 20/15. The patient's general health in adult life had been unremarkable except for a gonoccocal urethritis a year ago; this had responded rapidly to penicillin. Biomicroscopically the left cornea showed 'subepithelial dot-like infiltrates and diffuse clouding of the superficial stroma while a superficial pannus extended inward $2 \mathrm{~mm}$ from the limbus'. There was no vital staining of the cornea. Keratitis of unknown

Correspondence to Professor B. W. Streeten, Department of Ophthalmology, Upstate Medical Center, Syracuse, NY 13210, USA. cause, possibly viral, was suspected. Topical treatment with tetracycline and hydrocortisone eye ointment produced no improvement. The patient refused biopsy.

Over the intervening years symptoms of irritation subsided, but there was a gradual deterioration in vision of the left eye, which by October 1977 was $20 / 100$, not improved with spectacles or contact lenses. Biomicroscopy of the affected cornea at this stage revealed a greyish ground-glass appearance of the epithelium and superficial stroma. The corneal epithelium and adjacent conjunctiva were studded with myriads of white dots of various diameter which were just visible at $\times 20$ magnification (Fig. 1A). Corneal sensation was normal and there was no vital staining.

An $8 \mathrm{~mm}$ perforating graft of the affected left cornea was undertaken. Histological examination of the host disc showed severe dysplasia of the corneal epithelium alternating with areas of carcinoma-in-situ. This diagnosis stimulated further examination of the left eye, when it was observed that there was widespread thickening of the bulbar conjunctiva terminating abruptly some $5-8 \mathrm{~mm}$ from the limbus. In the affected conjunctiva a fine papillary or vertical 'corkscrew' type of neovascularisation was rendered more obvious by the general postoperative congestion (Fig. 1B). Multiple biopsies confirmed the extent of conjunctival involvement delineated with the slit-lamp.

After several weeks the corneal graft became covered with epithelium which resembled the host tissue in that it was translucent and contained many small white dots. An attempt to remove the affected epithelium was made. The corneal epithelium was 

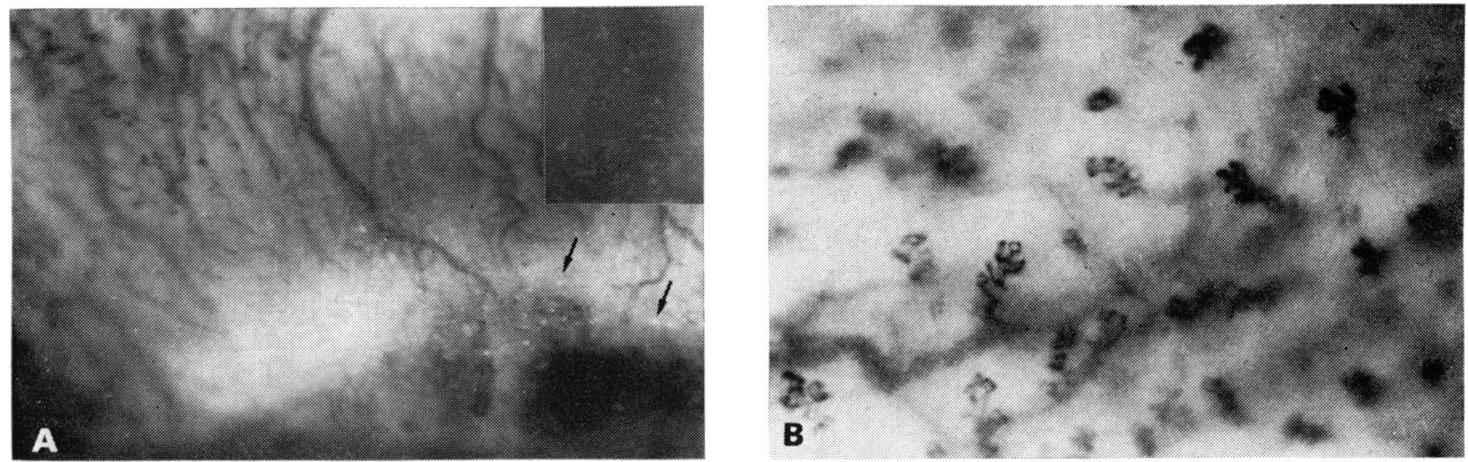

Fig. 1 Case 1. A. Corneoscleral junction. Microcysts appear as myriads of white dots (arrows) in carcinomatous epithelium. Black dots at upper left are vertical new vessels seen end on. Inset shows microcysts in thickened corneal epithelium. B. Detail of conjunctival new vessels which have a frond-like configuration.

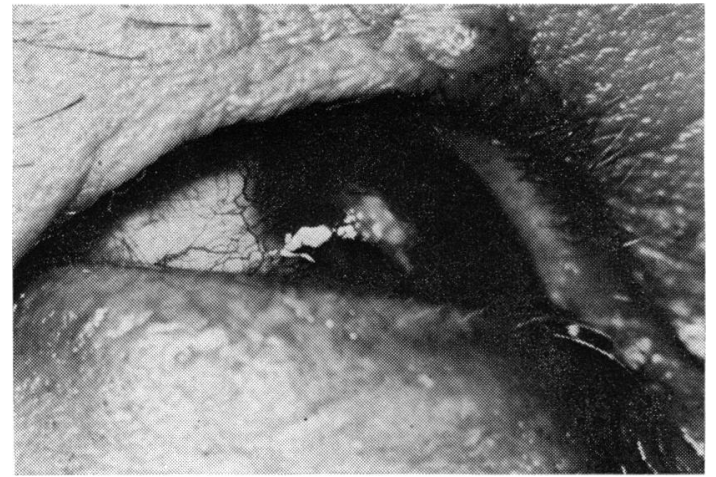

Fig. 2 Case 2. White plaque-like and smaller dot opacities superficially in a translucent, avascular, preinvasive carcinoma of the cornea.

dried and then liberally painted with absolute alcohol, followed by debridement. The diseased conjunctiva with a few millimetres of apparently healthy tissue was excised, but probable residual tumour was shown in 3 quadrants microscopically. Three weeks later the denuded area was covered by regenerated epithelium which was translucent, thickened, and again contained white dots. Biopsy indicated recurrence of the dysplasia/carcinoma-insitu. Observation over the past 2 years has shown no clinical change.

\section{CASE 2}

A 52-year-old white male in excellent general health was first seen by us in January 1970 . He stated that a greyish lesion of the right cornea had been present for the past 2 years. The condition had begun with severe irritation and blurred vision initially diagnosed as a viral keratitis. After the initial acute episode the greyish central corneal lesion had not changed much until recently, when it became noticeably raised. His ophthalmologist had noticed that the lesion contained white dots of varying size, some of which were visible to the naked eye. These he had considered as calcific plaques and had removed them from time to time. Over the past 3 months the lesion had become raised and extended toward the inferotemporal limbus (Fig. 2). Biopsy revealed a well differentiated squamous carcinomain-situ of the corneal epithelium. Excision of the tumour by lamellar keratectomy together with removal of the adjacent limbal conjunctiva and Tenon's capsule was undertaken. The tumour did not recur. The patient died from coronary thrombosis 3 years later.

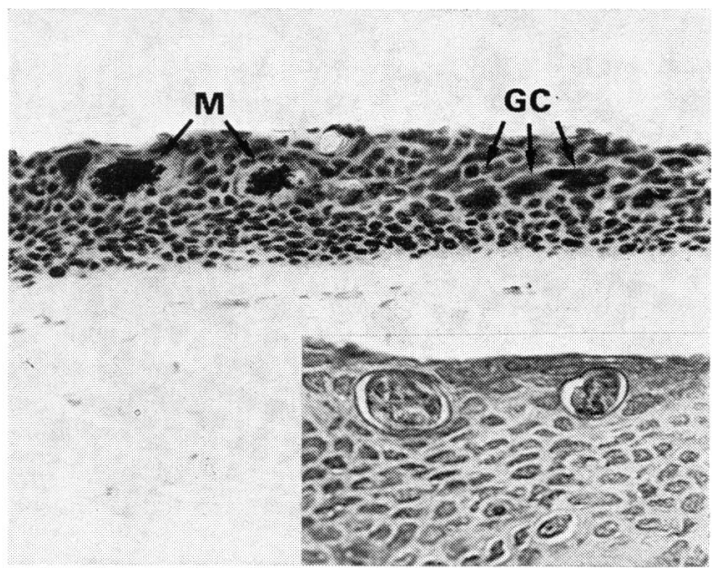

Fig. 3 Case 1. Carcinoma-in-situ of the corneal epithelium showing 3 large epithelial giant cells $(G C)$, and two microcysts $(M)$ containing dense basophilic material. $(H \& E, \times 175)$. Inset: Microcysts are lined by Alcian-blue-positive material like that of the superficial squames. $(\times 295)$. 


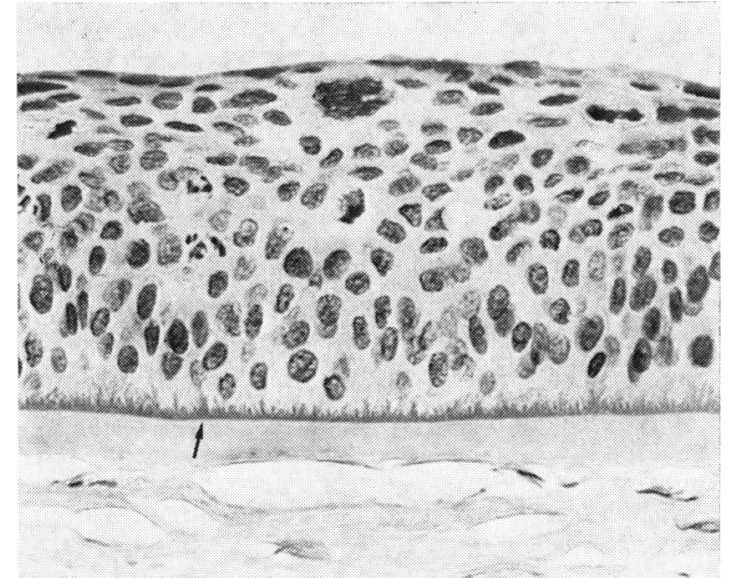

Fig. 4 Case 1. Fern-like extensions of the basement membrane (arrow) between the basal corneal epithelial cells. An epithelial giant cell is present near the surface centrally. $(P A S, \times 433)$.

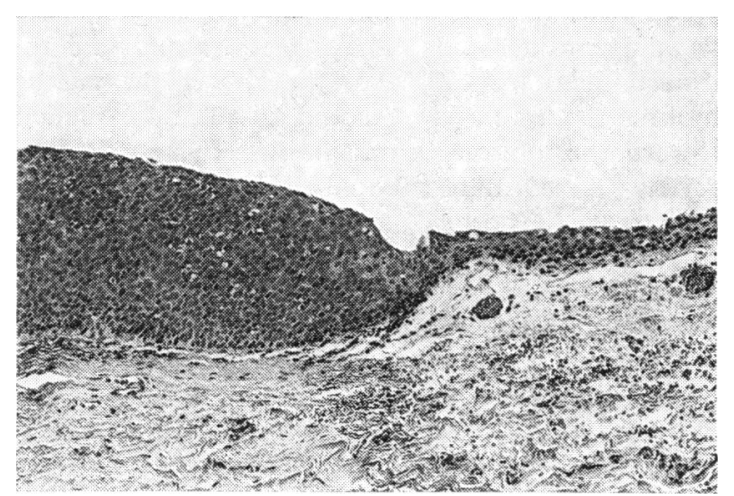

Fig. 5 Case 1. Bulbar conjunctiva showing abrupt junction of carcinoma-in-situ with normal conjunctiva. $(H \& E, \times 96)$.

\section{Methods and materials}

The 2 keratoplasty specimens were fixed in $10 \%$ neutral buffered formalin and divided. Half of each was embedded in paraffin for light microscopy and routine histochemistry. The other halves were osmified, embedded, and sectioned in epoxy resin prior to transmission electron microscopy. Conjunctival biopsies from case 1 were treated similarly.

\section{Results}

\section{LIGHT MICROSCOPY}

Case 1. The corneal disc showed abnormalities which were confined to the epithelium and superficial stroma. The epithelium was hyperplastic, the normal 5 layers being increased to 12 or more. Areas of dysplasia in which loss of basal polarity was a prominent feature alternated with areas of carcinoma-in-situ showing hyperchromatism, epithelial giant cells, pleomorphism, and abnormal mitoses (Fig. 3). Numerous microcysts were present in all areas of the epithelium. Several types were evident. Cysts of 40-100 $\mu \mathrm{m}$ contained debris which stained with Alcian blue, colloidal iron, and periodic acid Schiff (PAS). Smaller cysts contained a single shrunken densely stained cell. Finally, there were small cystic spaces which appeared almost empty apart from fine granular aggregates. In most sections vertical tufts of basement-membrane-like

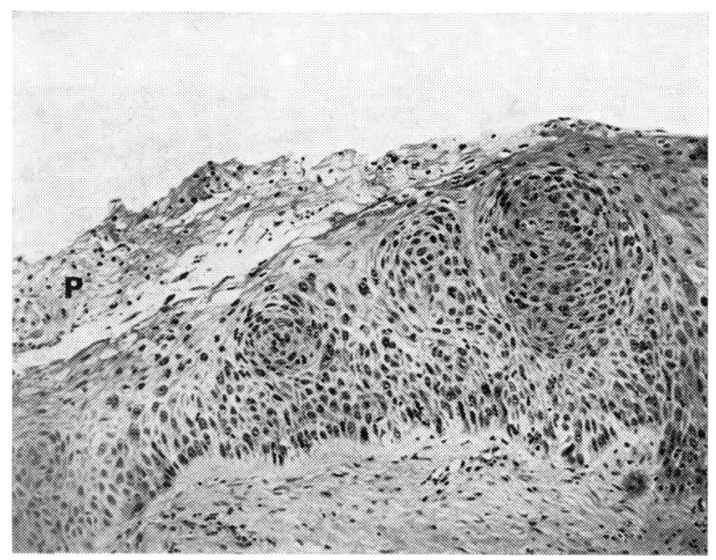

Fig. 6 Case 2. Carcinoma-in-situ of corneal epithelium showing large epithelial whorls and patch of parakeratosis $(P)$. There is pannus under the epithelium, but the basement membrane is intact. $(H \& E, \times 124)$.

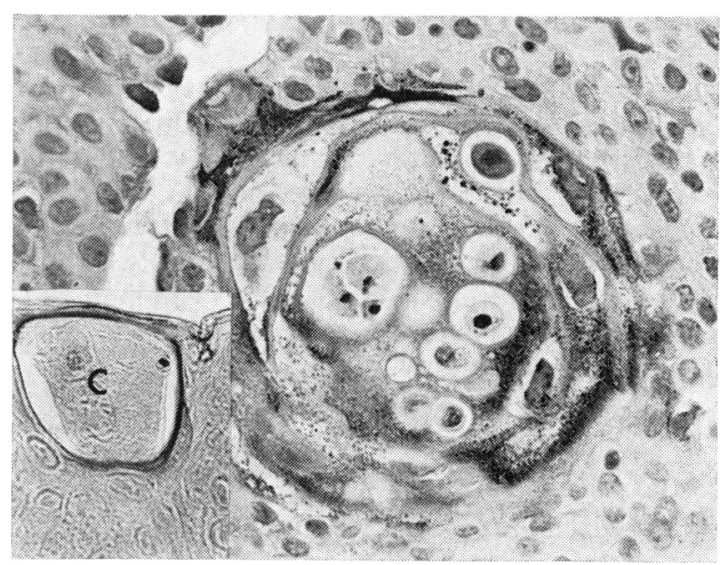

Fig. 7 Case 2. Corneal epithelium showing large dyskeratotic whorl containing many microcysts. $(H \& E, \times 337)$. Inset: Wall of microcyst $(C)$ staining with Alcian blue. $(\times 293)$. 
material extended into the basal cell layer (Fig. 4). There was no evidence of extension of the epithelial cells through the basement membrane. No subepithelial inflammatory cells were present, but there was minimal anterior stromal scarring.

The conjunctival biopsies showed similar changes, but in addition capillaries with a minimum of con- nective tissue extended from the lamina propria into the epithelium in the limbal area. The transition to normal conjunctiva at the edges of the lesion was characteristically abrupt (Fig. 5) with a tendency for the carcinoma to override the normal epithelium.

Case 2. The corneal epithelium showed changes

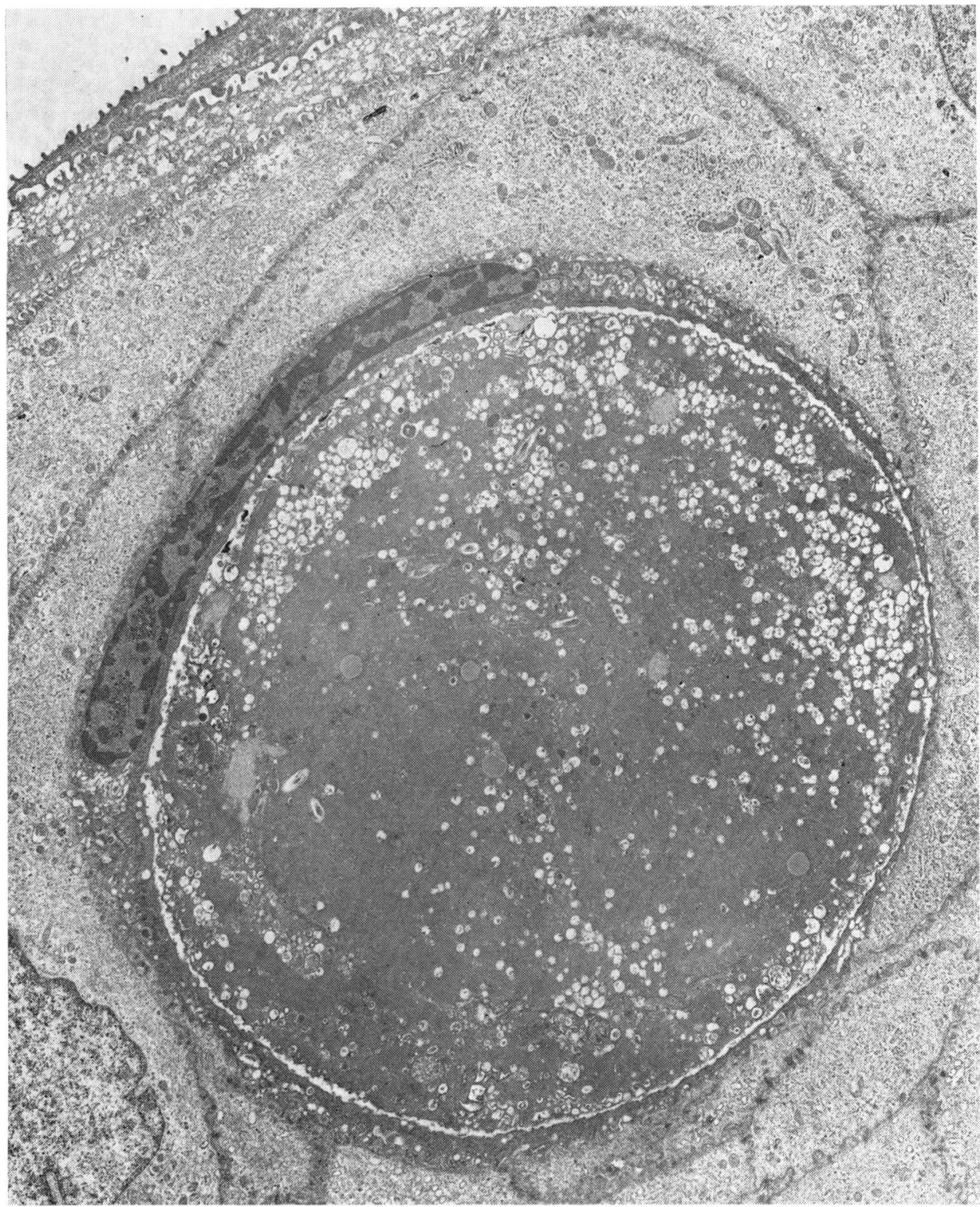

Fig. 8 Case 1. Large microcyst. Lining shows few microvilli. Cyst contents include vacuolated electron-dense material and other cell debris. Adjacent cells contain some increase in mitochondria, endoplasmic reticulum, and tonofibrils for this layer. $(T E M, \times 6160)$. 
of keratinising carcinoma-in-situ. The grossly disordered epithelium showed numerous mitoses as well as occasional giant cells, dyskeratotic whorls of cells, and parakeratosis (Fig. 6). Nests of dyskeratotic cells, some of which were encysted (Fig. 7), were regularly seen. Small microcysts similar to those in case 1 were present (Fig. 7, inset) but not frequent. Bowman's layer, although absent in places, was not invaded by tumour, and the basement membrane appeared intact. In some areas a layer of loose fibrillar material often showing a striated appearance was interposed between Bowman's layer and the basement membrane (Fig. 13, inset A). This material stained poorly with PAS but was strongly coloured with Alcian blue. The stroma underlying the tumour was moderately vascularised and infiltrated with lymphocytes and occasional plasma cells. Capillaries from this layer extended vertically into the tumour.

ELECTRON MICROSCOPY

The cancerous epithelium in both cases contained an excessive number of organelles, including mitochondria, endoplasmic reticulum, and tonofilaments (Fig. 8). There appeared to be a reduction in desmosomes, which in places had become invaginated or engulfed and lay free within the epithelial cell cytoplasm (Fig. 9).

Microcysts containing disintegrating cytoplasmic debris and organelles were particularly frequent in case 1 (Fig. 8). The cyst walls were formed by the cell membranes of adjacent cells which had become flattened and wrapped around the contents. The lining membrane thus formed was often wavy in outline but rarely showed well formed microvilli. Occasionally disintegrating cells had been engulfed by epithelial phagocytes without a surrounding cell membrane (Fig. 10). In case 1 the neoplastic epithe-

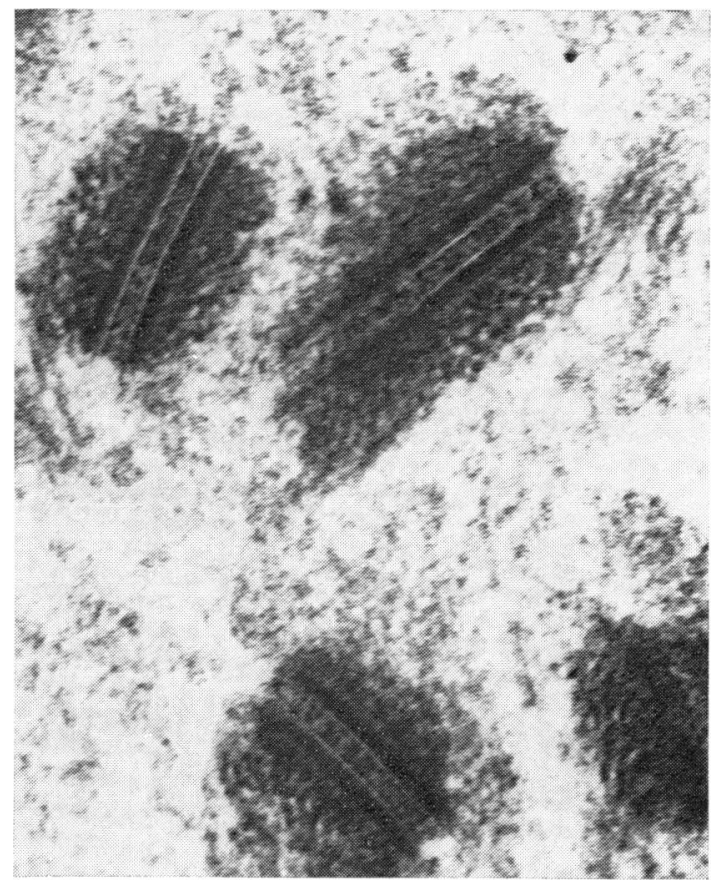

Fig. 9 Case 1. Cluster of desmosomes lying free within cytoplasm of corneal epithelial cell. $($ TEM,$\times 20816)$.
Fig. 10 Case 1. Corneal epithelial cell has engulfed a disintegrating cell. (TEM, $\times 4955)$.

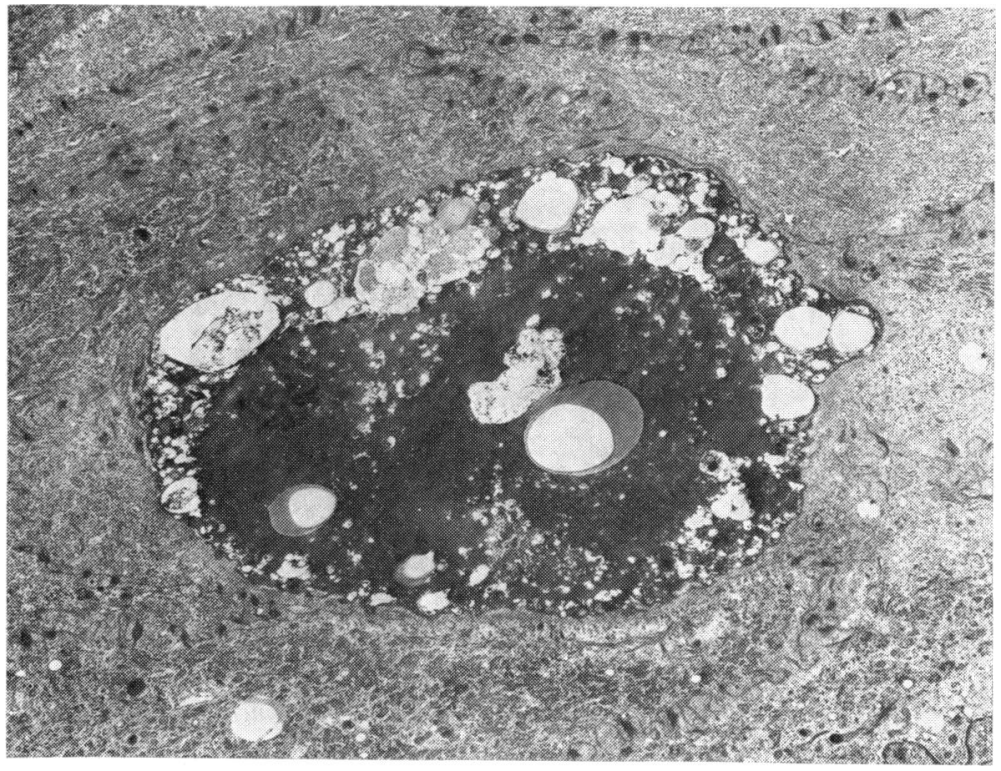


lial cells of the middle layer often contained large cytoplasmic vacuoles in which dense amorphous material intermixed with fibrils $14-16 \mathrm{~nm}$ wide with microbanding at $3 \mathrm{~nm}$ were found (Fig. 11). In some of these vacuoles the amorphous material was arranged in circumferential bands with the fibrils lying in groups of 2-6 parallel to each other and perpendicular to the amorphous material; in others both elements were unoriented.

Basement membrane alterations were prominent
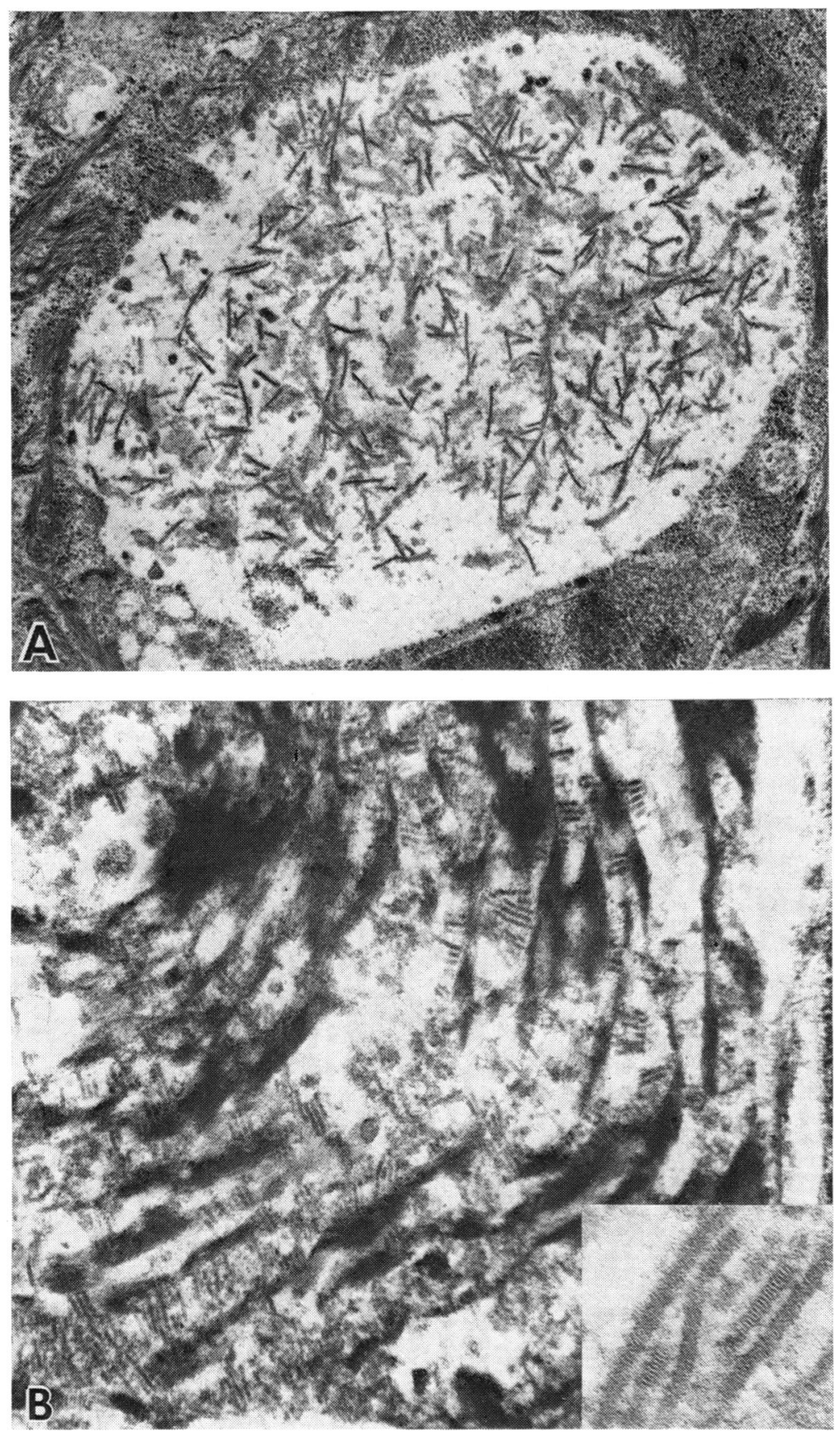

Fig. 11 Case 1. Corneal epithelium. Intracytoplasmic vacuoles containing amorphous material mixed with fibrils. In $A$ these 2 elements are arranged randomly. $(T E M, \times 24000)$. In $B$ the fibrils are parallel to each other and lie perpendicular to the amorphous material, which is disposed circumferentially. (TEM, $\times 37$ 612).

Inset $B$ : The fibrils are banded at $3 \mathrm{~nm}$. (TEM, × 177672$)$. 
in both patients. In case 1 a layer of amorphous granular material was found between the basement membrane and Bowman's layer. At regular intervals the basement membrane was thrown into vertical frond-like folds (Fig. 12) corresponding to those seen by light microscopy (Fig. 4). Besides granulomatous material, the core of these protrusions contained collagen-like fibres with coarse banding at $55 \mathrm{~nm}$ and a microperiodicity of $9 \mathrm{~nm}$. In case 2 similar fibrillogranular material sometimes con-

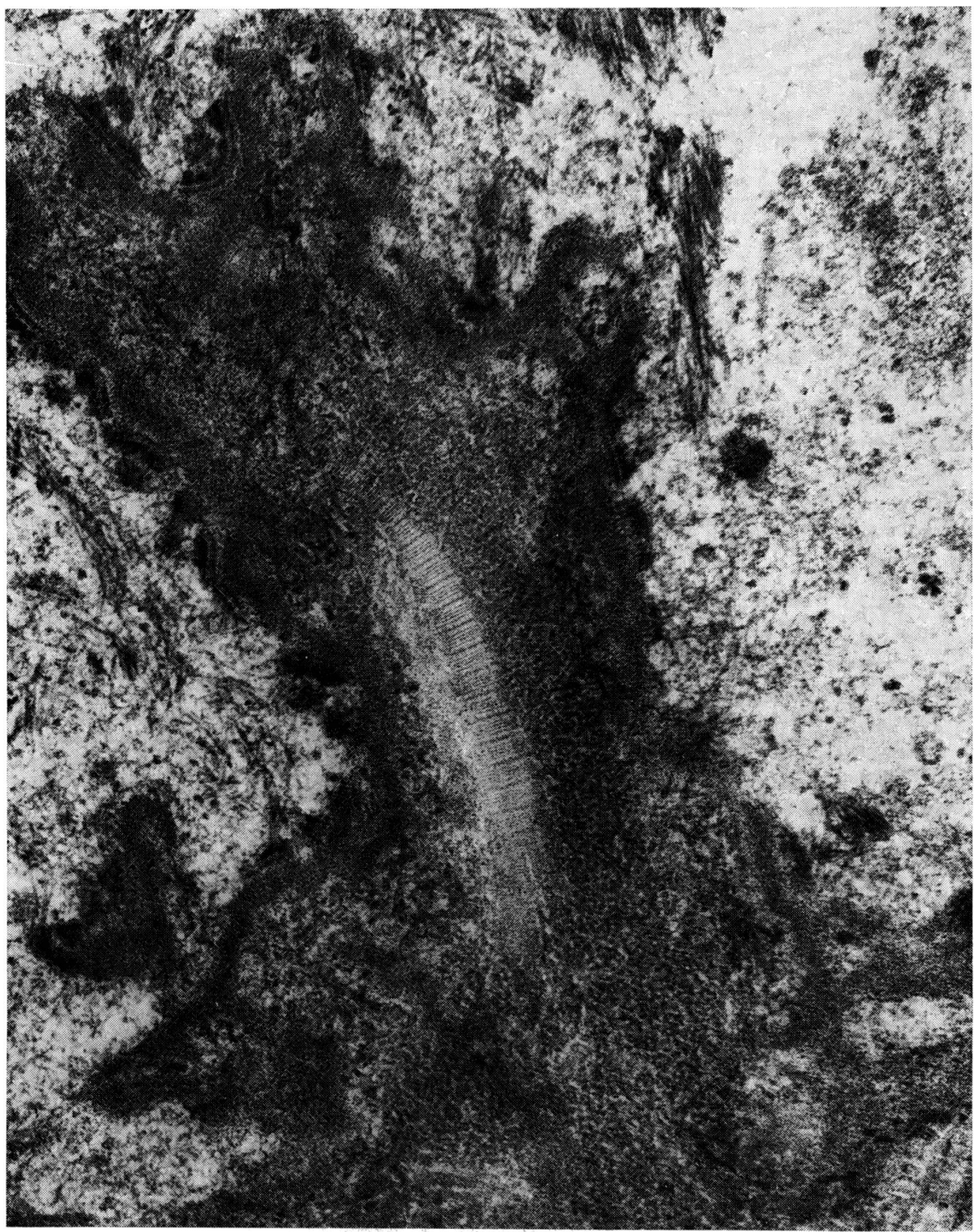

Fig. 12 Case 1. Corneal basement membrane is thrown into vertical folds by accumulation of abnormal fibrillogranular material beneath it. Folds often contain prominent fibrils banded at $55 \mathrm{~nm}$ as here. $($ TEM, $\times 59210$. 


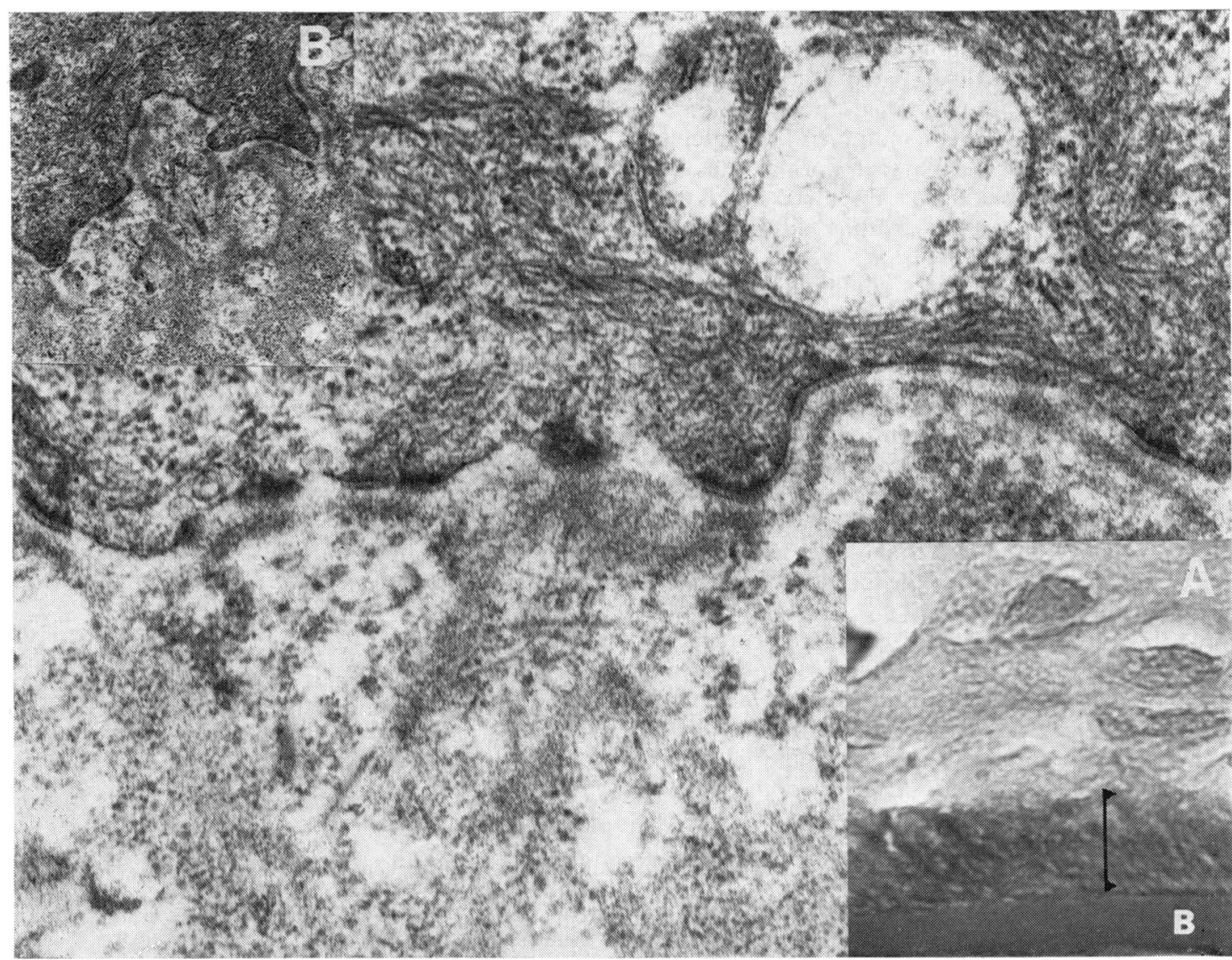

Fig. 13 Case 2. Corneal epithelial basement membrane rests on a fibrillogranular layer of abnormal material.

(TEM, $\times 51$ 280). Inset A: By light microscopy this layer (bracket) has an oblique striated appearance. B=Bowman's membrane. $(H \& E, \times 977)$. Inset B: Processes of the basal cells extend irregularly into this thick layer.

$(T E M, \times 17337)$.

taining dense bodies and vacuoles was interposed between the basement membrane of the irregular basal epithelium and Bowman's layer (Fig. 13).

\section{Discussion}

This report concerns 2 cases of carcinoma-in-situ of the cornea and in 1 case also of the bulbar conjunctiva. There was neither clinical nor microscopic evidence of inflammation at the time we studied them, although an acute superficial keratitis had given rise to the initial symptomatology in both patients. The biomicroscopic appearances, somewhat vaguely recorded, had suggested a viral aetiology to their ophthalmologists. Such inflammatory episodes occurring as a prelude or accompaniment of carcinoma of the conjunctiva have been often noted in the past. Thus Irvine ${ }^{1}$ reported 3 cases of diffuse growth involving the bulbar conjunctiva (not unlike our first patient) in whom marked inflammation obscured their carcinomatous nature until conjunctival smears and biopsies were examined. It is of interest that 1 of these patients later developed regional metastases.

White dots were present in the lesions seen in both our cases. In the first patient they were visible biomicroscopically as myriads of white, epithelial punctae which did not stain with vital dyes. Microscopically they consist of microcysts and vacuoles of various sizes, scattered throughout the corneal and bulbar conjunctival epithelia. They appear to originate from disintegrating cells which have been subsequently enveloped by adjacent cells without actually being phagocytosed. In this respect they resemble epithelial microcysts seen in a variety of unrelated disorders which have been described by 
Tripathi and Bron. ${ }^{2}$ The plasmalemmal lining of the microcysts seen in our patient, however, was smooth or only slightly crenated in contrast to the prominent microvillous configuration seen in the microcysts found in other conditions. This difference may relate to the tendency for normal cell interdigitations to be lacking or absent in this type of carcinoma as noted by Tripathi, ${ }^{3}$ although it was not observed in our patients.

Intracytoplasmic vacuoles containing amorphous electron-rense material together with peculiar fibrils showing banding at $3 \mathrm{~nm}$ were regularly seen in the midepithelial layers of the first patient. Their origin is not known. Possibly they result from degradation and repolymerisation of fibrous proteins such as compose the tonofibrillar system, which was excessively developed in many cells. Vacuoles of this type were not observed in the second case, though there were occasional microcysts of degenerating cells. It may be deduced that the macroscopic white plaques seen in the second patient probably represented the extensive patches of parakeratosis observed microscopically. The presence of clusters of desmosomes dissociated from their parent cell membranes and lying free in the cytoplasm of epithelial cells was an occasional finding in both patients. This phenomenon was noted in Bowen's disease of the skin by Seiji and Mizuno $^{4}$ as well as in preinvasive carcinoma of the cornea by Tripathi and Garner. ${ }^{3}$

Prominent ultrastructural changes which do not appear to have been previously noted in this condition were present in relation to the basement membrane of the corneal epithelium. In both cases a layer of fibrillogranular material was deposited between the basement membrane and Bowman's layer. In the first patient this substance covered by the basement membrane extended upwards as frond-like protrusions into the epithelium. A similar layer was seen in the second patient, but interepithelial projections were not observed. It is probable that this basement-membrane-like material originates from the disordered epithelium. Microcysts of different types, together with abnormal basement membrane material, seem to represent the response of the corneal epithelial to a variety of unrelated stimuli. ${ }^{5}$

The junction between apparently normal epithelium and the thicker dysplastic or carcinomatous tissue was abrupt in both patients. This well recognised finding provides a means by which the limits of the tumour may often be determined in optical section of the slit-lamp. However, the diseased epithelium is sometimes only minimally thickened, ${ }^{6}$ so that the precise limits of bulbar extension must always be determined histologically by multiple punch biopsies. Cicatricial distortion of the conjunctival vessels at the healed biopsy sites permits their ready identification prior to definitive surgery or radiotherapy, or, when it is indicated, to further biopsies from more peripheral sites.

Miss Sharon Edwards was responsible for the technical aspects of microscopy in this report.

This study was supported in part by the Medical Research Service of the Veterans Administration Hospital, grant EY01602 from the National Institutes of Health, as well as the British National Institute for Prevention of Blindness.

\section{References}

IIrvine RA. Diffuse epibulbar squamous-cell epithelioma. Am J Ophthalmol 1967; 64: 550-4.

${ }^{2}$ Tripathi RC, Bron AJ. Cystic disorders of the corneal epithelium. II. Pathogenesis. Br J Ophthalmol 1973; 57: 376-90.

${ }^{3}$ Tripathi RC, Garner A. The ultrastructure of preinvasive cancer of the corneal epithelium. Cancer Res 1972; 32: 90-7. ${ }^{4}$ Seiii M, Mizuno F. Electron microscopic study of Bowen's disease. Arch Dermatol 1969; 99: 3-16.

${ }^{5}$ Dark AJ. Cogan's microcystic dystrophy of the cornea: ultrastructure and photomicroscopy. $\mathrm{Br} \mathrm{J}$ Ophthalmol 1978; 62: 821-30.

${ }^{6}$ Zimmerman LE. In: Boniuk M, ed. Ocular and Adnexal Tumors. Saint Louis: Mosby, 1967: 60. 